\title{
CNN Based Analysis of COVID-19 Using Chest X-Ray Images
}

\author{
Soham Taneja ${ }^{1}$, Dr. Meenu Gupta ${ }^{2}$, Dr. Rachna Jain ${ }^{3}$ \\ ${ }^{1,3}$ Department of CSE, Bharati Vidyapeeth's College of Engineering, Delhi, India \\ ${ }^{2}$ Department of CSE, Chandigarh University, Punjab, India \\ Email: sohamtaneja12@gmail.com,gupta.meenu5@gmail.com, \\ rachna.jain@bharatividyapeeth.edu
}

\begin{abstract}
:
Coronavirus (COVID - 19) is a deadly virus that originally originated from China's Wuhan district around November last year. It has a deadly effect on the human respiratory system if the condition escalates. Currently, millions of people have been affected worldwide, and in countries like India, the cases are still on the rise. Due to an increased rise in cases, the testing facilities are struggling to keep up with the demand for testing, and medical experts are looking for alternate ways to speed up testing. In this paper, we have experimented with one such way by developing a CNN-based model to classify the chest X-ray images for the detection of coronavirus affected cases. For result analysis, we have applied CNN based VGG 16, VGG 19, and custom model. Further, we compare the result of these models based on accuracy. In this experimental analysis, VGG 19 model detected 99\% of COVID-19 infected cases accurately as compared to VGG 16. This is entirely an experimental study and should not be used in real-life scenarios without an evaluation by medical experts and determining the effectiveness of this method.
\end{abstract}

Keywords: Covid-19, Deep-learning, CNN, VGG-16, VGG-19, Chest X-ray images

\section{Introduction}

Coronavirus is a deadly mutated version of SARS, which emerged this year and caused worldwide panic and disruptions [12]. With the increasing number of cases and limited medical staff, the testing speed and capacity gets restricted. CNN based models are assumed to be extremely efficient for the task of image classification [10] [11]. Pertained models are loaded first before starting to train the entire model. Doing so, the model can extract low-level features from the given input images and hence, achieves greater accuracy in less number of epochs, reducing the training time. In [1], the authors have drawn a parallel between COVID-19 and SARS and MERS. They have also analyzed the chest X-rays for different conditions. In [2], the authors have used transfer learning to classify the chest X-ray scans using CNNs. They have achieved $96.78 \%$ accuracy in doing so. Further, in [3], the authors have used a deep learning-based CNN model to classify COVID-19 chest X-rays using a dataset consisting of 225 positive cases.

With this paper, we aim to develop a theoretical CNN-based model to predict the positive and negative coronavirus cases using chest $\mathrm{X}$-ray scans of patients. We have drawn a parallel between the classification performance of our $\mathrm{CNN}$ model with the popular models (VGG-16 and
VGG-19). In our results, we inferred that the VGG19 model performed the best with $99 \%$ accuracy. However, we also assume the model to be overfitting slightly, and our assumption can be clarified when more data of COVID patients is made public in the future. This paper, further classified in the different sections such as section 2 , discussed the dataset used, and model formulation. Different matrices and algorithms used are also discussed in section 2. Further, the conclusion and future scope are discussed in section 3 .

\section{Related Work}

Many types of research are being done on the prediction and analysis of COVID-19 using Chest x-ray images. Here are some of the researches listed below from all over the world.

In [13], A. Narin et al. worked on three distinct convolutional neural network frameworks, namely, Inception v3, Inception-Resnet v2 and Resnet50 for the analysis of pneumonia in coronavirus patients utilizing the chest $\mathrm{x}$-ray images. The pre-trained Resnet50 model gives the best result as compared to the other two models. In [14], A. Borghesi et al. worked on an experimental chest $\mathrm{x}$-ray scoring system which is applied on the COVID-19 pneumonia patients who are hospitalized, to gauge the severity and advancement of this particular 
disease. The outcomes of preliminary validation are also depicted on 100 hospitalized patients with SARS-Cov-2 disease.

Next in [15], R. Pereira et al. proposed a schema of hierarchical, multi-class classification as pneumonia can be figured as a hierarchy. The utilization of resampling algorithms is also proposed for the sake of rebalancing the distribution of classes. The textures were found out to be the major visual attribute of the $\mathrm{x}$-ray scans and a pre-trained CNN model was employed for obtaining the results. Next in [16], P. Afshar et al. presented an alternative framework which is based on capsule networks also called COVID-CAPS and is mostly used for small datasets. The results obtained depicted that this model had better results (which also included fewer parameters to be trained) than the previous CNN models. The pretraining with a similar kind of dataset raised the accuracy to $98.3 \%$ in this scenario.

Further in [17], T. Ozturk et al. proposed a deep model for the detection of COVID-19 causalities by the use of x-ray images. An accuracy of $98.08 \%$ was obtained for the binary classes whereas $87.02 \%$ obtained for the multi-class. The heatmaps that were depicted are an asset to locate the particular regions which are affected by the $\mathrm{x}$-rays.

Next in [18], N. S. Punn and S. Agarwal et al. presented the random oversampling along with the weighted class loss function method for the unbiased fine-tuned learning in several deep learning models like Inception v3, Densenet169 and NASNetLarge for the binary and multi-class classification of posteroanterior chest $\mathrm{x}$-ray images. The NASNetLarge depicted the best scores as compared to other architectures. In [19], A. Abbas et al. adapted a deep CNN model known as Decompose, Transfer and Compose, combinedly named as DeTraC for the classification of COVID19 chest $\mathrm{x}$-ray images. The model proposed was able to deal with irregularities by utilizing the class decomposition mechanism in the dataset. An accuracy of $95.12 \%$ was achieved by this model in performing the classification.

In [20], M. Karim et al. worked on an explainable deep neural network for the automated detection of the symptoms of COVID-19 through the $\mathrm{x}$-ray images. The number of x-ray images used were 15,959 in total and were preprocessed, augmented followed by classification through an ensemble method and emphasizing of class-discriminating areas. Along with this, human interpretable results were also provided. This study would help the application process in clinical practice.

Further in [21], S. Minaee et al. worked on deep learning applications for COVID analysis using $\mathrm{x}$ ray images. A dataset of 5000 images was made from already existing datasets and transfer learning was applied for the training of four CNN models that were Resnet18, Resnet50, SqueezeNet and DenseNet121 for the detection of COVID-19. Heatmaps were also generated to study the lung regions of the infected patients.

Later in [22], X. Li et al. presented a light DNN based application that was used for taking the snaps of the chest $\mathrm{x}$-ray images for the screening of COVID-19. A novel 3 player knowledge transfer and distillation model including a trained physician used for extracting features from x-ray images were designed. A novel loss function along with training schemes was employed for the noisy images to grasp the imaging features for correct on device screening.

After discussing the various researches, this study is focused on the Chest X-Ray Images analysis for evaluating the impact of COVID-19 using CNN.

\section{Materials and Methods}

The dataset used and the methodology used is explained in the subsequent sections

\subsection{Dataset}

The dataset for this paper was obtained from two sources (i.e., Github [7] and Kaggle [8]). This collected dataset is not meant to claim the diagnostic ability of any Deep Learning model but, to research about various possible ways of efficiently detecting Coronavirus infections using computer vision techniques.

We used 133 PA X-ray images of infected patients along with $150 \mathrm{X}$-ray images of healthy patients to train our model. We reshaped the images to $(256,256)$ to reduce training time. The data is currently limited as it is not released in the public domain yet. The distribution of images for training and testing is mentioned in Table 1 below:

Table 1: Dataset Distribution

\begin{tabular}{|c|c|c|}
\hline & Train & Tests \\
\hline Healthy Person & 127 & 23 \\
\hline Covid-19 Infected Patient & 113 & 20 \\
\hline
\end{tabular}




\subsection{Model Formulation and Results}

The experimental results are calculated based on metrics used, such as accuracy, precision, and recall [6].

\subsubsection{VGG-16}

It is a simple CNN model developed by the Visual Geometry Group from Oxford [9]. We chose this model specifically to avoid choosing complex models, like Resnet of Inception net, since our training set is small. Figure 1 depicted the architecture for the VGG-16 model, which is used in an abstracted form.

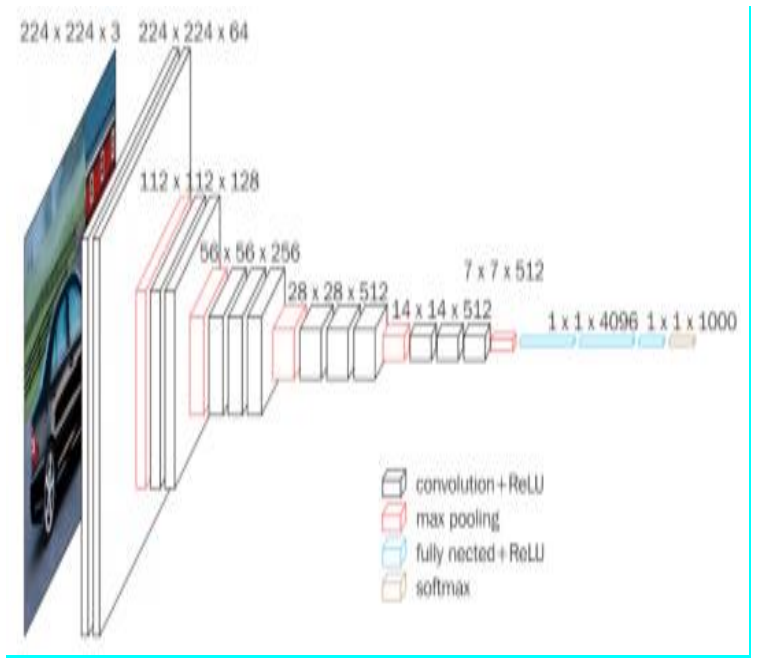

Figure 1: Abstract form of VGG 16 Model [4]

In this model, the collected dataset of healthy and COVID-19 X-ray image is trained and tested. In training, the result of Sensitivity is 0.954 , and Specificity is 0.982 , as shown in table 2 .

Table 2: Training dataset

\begin{tabular}{|l|l|l|l|}
\hline Label & Precision & Recall & f1-score \\
\hline Normal & 0.95 & 0.98 & 0.97 \\
\hline Covid - 19 & 0.98 & 0.95 & 0.96 \\
\hline Accuracy & & & 0.97 \\
\hline
\end{tabular}

Table 3: Testing Data

\begin{tabular}{|l|l|l|l|}
\hline Label & Precision & Recall & f1-score \\
\hline Normal & 0.88 & 1.0 & 0.94 \\
\hline Covid - 19 & 1.0 & 0.85 & 0.92 \\
\hline Accuracy & & & 0.93 \\
\hline
\end{tabular}

In the case of the testing dataset, sensitivity is 0.885 , and Specificity is 1.0 in the result, as shown in table 3 .

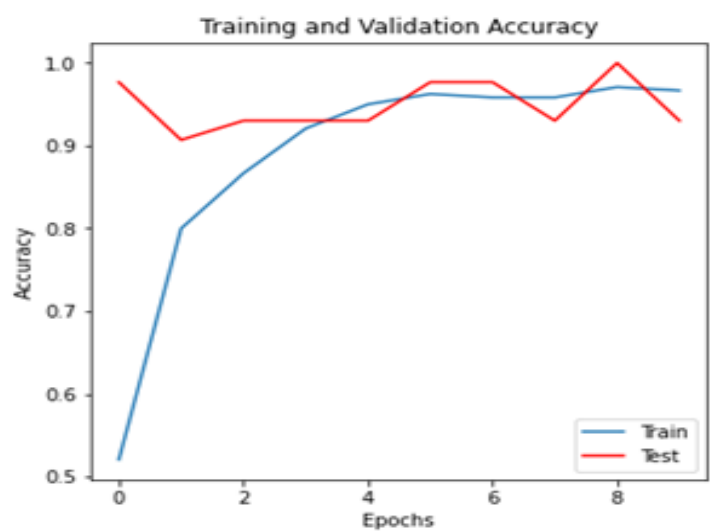

Figure 2 (a): Training and validation Accuracy of VGG-16

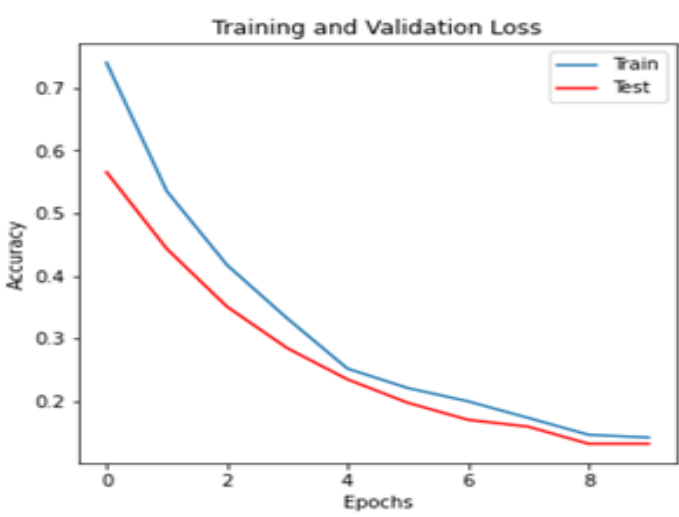

Figure 2 (b): Training and validation loss of VGG16

Figure 2 (a) shows the model accuracy for VGG 16 as it improves with the successive epochs, and figure 2 (b) shows the training loss for VGG 16 as it reduces with the consecutive epochs.

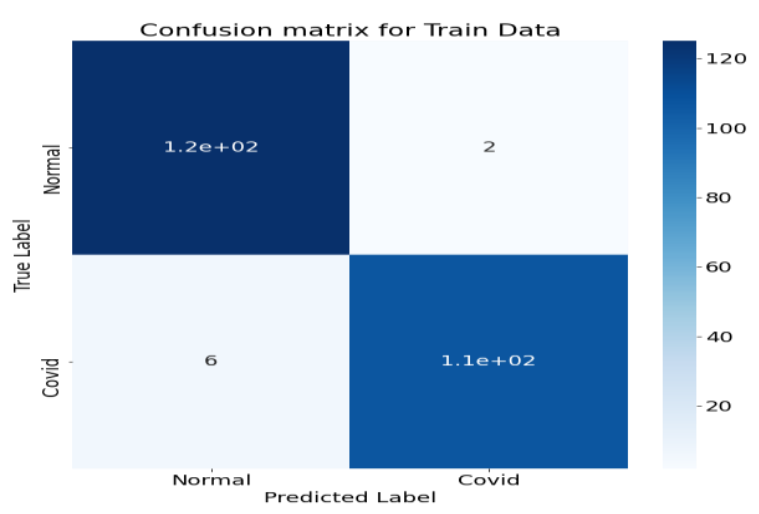

Figure 3 (a): Confusion matrix of train data of VGG-16 model 


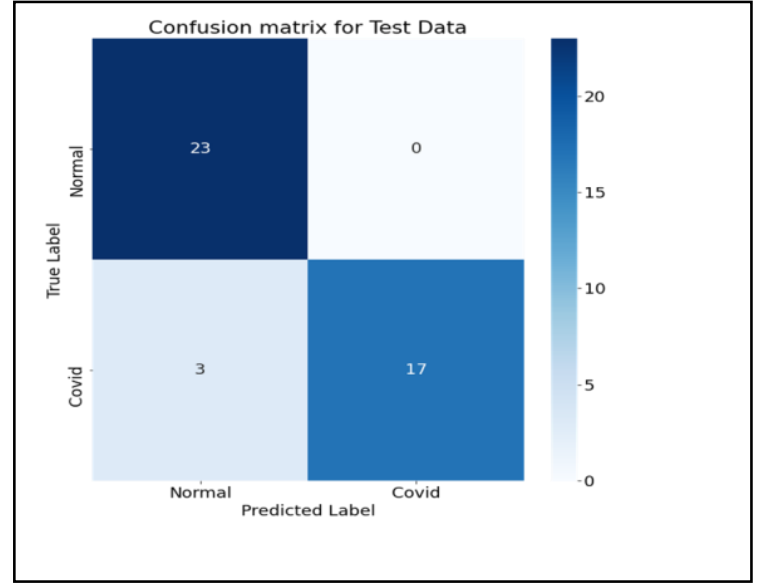

\begin{tabular}{|l|l|l|l|}
\hline Label & Precision & Recall & f1-score \\
\hline Normal & 1.0 & 1.0 & 1.0 \\
\hline Covid - 19 & 1.0 & 1.0 & 1.0 \\
\hline Accuracy & & & 1.0 \\
\hline
\end{tabular}

Figure 3(b): Confusion matrix for test data of VGG-16 model

Figure 3 (a) and figure 3 (b) show the confusion matrices obtained for the train and test set, respectively. Figure 3 shows that the misclassified samples are negligible.

\subsubsection{VGG-19}

It is a simple CNN model developed by Visual Geometry Group from Oxford, which is a variation of VGG-16. It has 19 deep layers, which are compared to 16 in VGG-16. This conversion helps to enhance the results obtained and enables better feature recognition. We chose this model specifically to avoid choosing complex models, like Resnet of Inception net, since our training set is small. Figure 4 depicted the architecture for the VGG-19 model, which is used in an abstracted form.

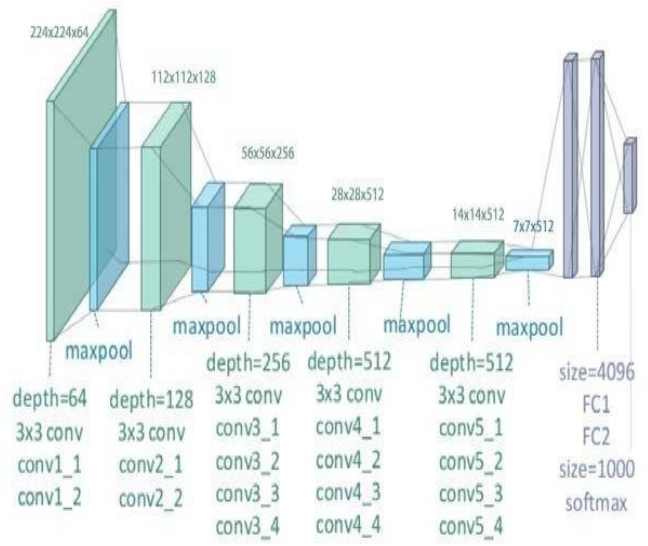

Figure 4: Abstracted form of VGG-19 model [5]
VGG-19 is a variant of the VGG model, which consists of 19 layers. In this model, 16 are convolutional layers where three are fully connected layer, 5 are MaxPool layers and one softmax layer. In this model, a collected dataset of healthy and COVID-19 X-ray image is trained and tested. In training, the result of Sensitivity is 1.0, and Specificity is 0.983 , as shown in table 6 .

Table 4: Testing Data

\begin{tabular}{|l|l|l|l|}
\hline Label & Precision & Recall & f1-score \\
\hline Normal & 1.0 & 0.98 & 0.99 \\
\hline Covid - 19 & 0.98 & 1.0 & 0.99 \\
\hline Accuracy & & & 0.99 \\
\hline
\end{tabular}

Table 5: Training data

In the case of the training dataset, sensitivity is 1.0, and Specificity is 1.0 in the result, as shown in table 5 .

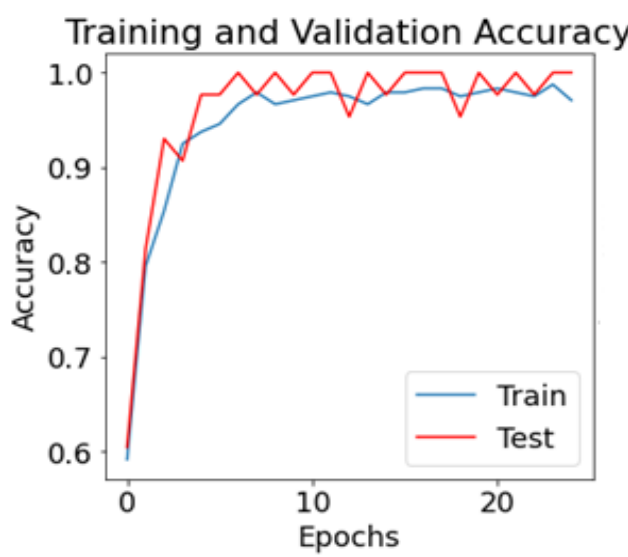

(a)

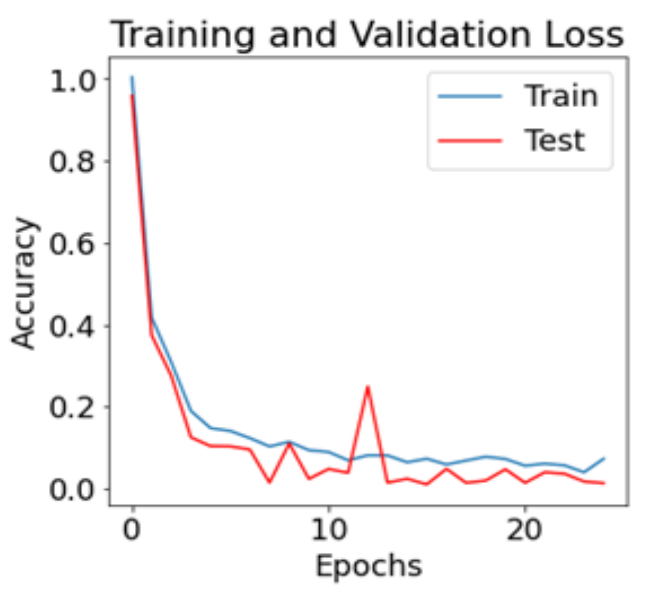

(b) 
Figure 5: (a) Training and validation Accuracy of VGG-19 model (b) Training and validation loss of VGG-19 model.

Figure 5 (a) shows the model accuracy for VGG 19 model as it improves with the successive epochs, and figure 5 (b) shows the training loss for VGG 19 model as it reduces with the successive epochs.

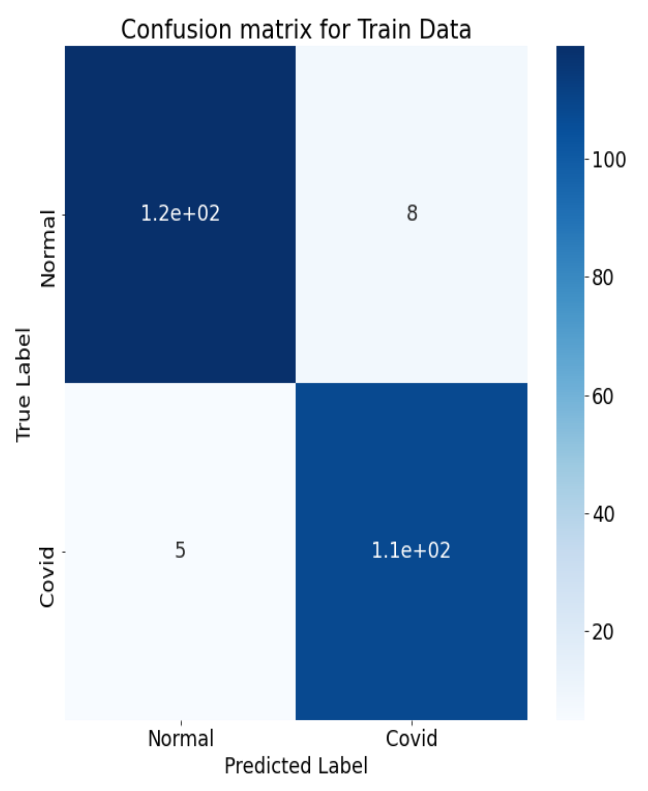

(a)

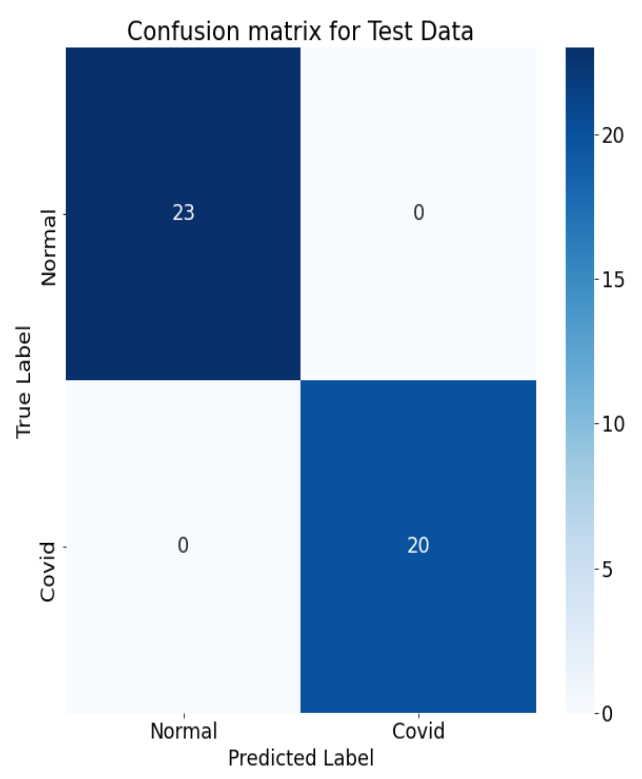

(b)

Figure 6: Confusion matrix of (a) train data (b) test data of VGG-19 model
Figure 6 shows the confusion matrices obtained for the train and test set, respectively. Figure 9 shows that the misclassified samples are negligible. The above figures 2,5 , and 8 show the training and testing performance as the model trains with successive epochs. We can see that our custom model is prone to over-fit, but it still gives optimal accuracy. Moreover, we can see that VGG 19 has the best performance among all the discussed models.

\subsubsection{Custom model}

In addition to the above-pre-trained models, we proposed a model to classify $\mathrm{x}$-ray scans. This model is shallower than the above two models but gives near about the same performance. This enables us to train our model faster and also reduces the inference time. Figure 7 represented the detailed architecture of our custom model used in this work.

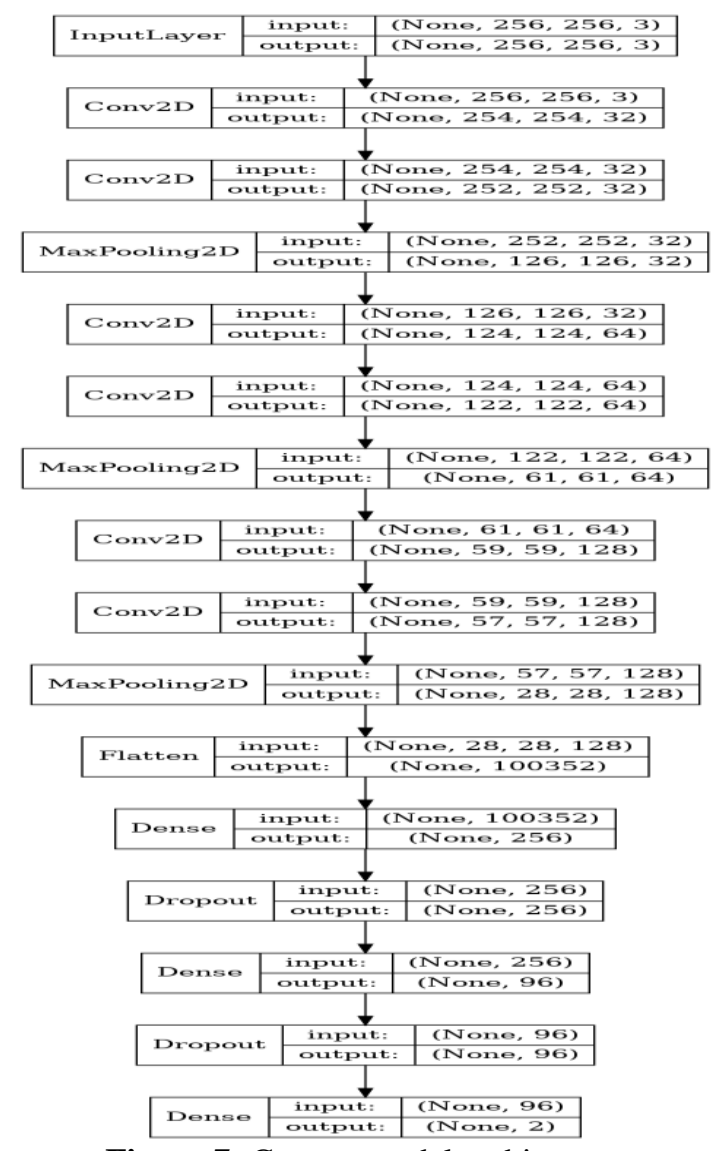

Figure 7: Custom model architecture

In this custom model, a collected dataset of healthy and COVID-19 X-ray image are trained and tested. In training, the result of Sensitivity is 0.96 , and Specificity is 0.93 , as shown in table 4 . 
Table 4: Training Data

\begin{tabular}{|l|l|l|l|}
\hline Label & Precision & Recall & f1-score \\
\hline Normal & 0.96 & 0.94 & 0.95 \\
\hline Covid - 19 & 0.93 & 0.96 & 0.94 \\
\hline Accuracy & & & 0.95 \\
\hline
\end{tabular}

Table 5: Testing Data

\begin{tabular}{|l|l|l|l|}
\hline Label & Precision & Recall & $\begin{array}{l}\text { f1- } \\
\text { score }\end{array}$ \\
\hline Normal & 1.0 & 1.0 & 1.0 \\
\hline Covid - 19 & 1.0 & 1.0 & 1.0 \\
\hline Accuracy & & & 1.0 \\
\hline
\end{tabular}

In the case of the training dataset, sensitivity is 1.0, and Specificity is 1.0 in the result, as shown in table 5 .

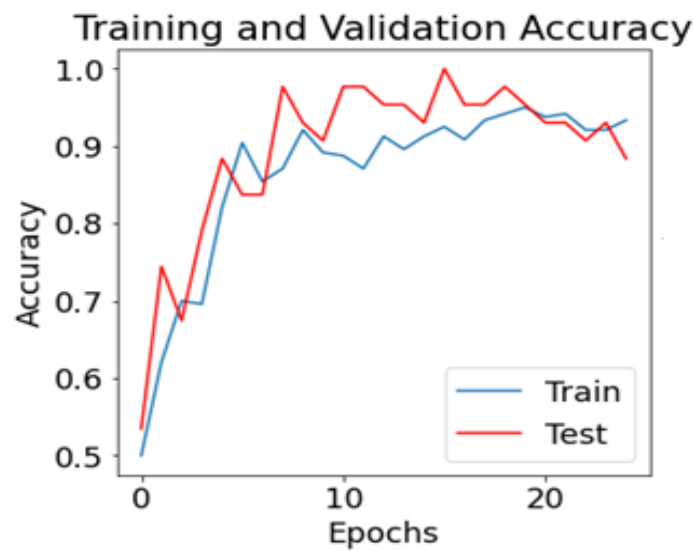

(a)

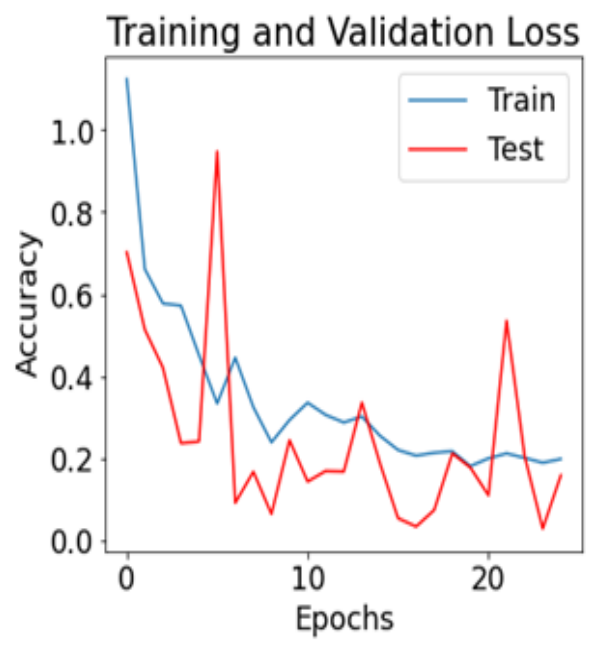

(b)
Figure 8: (a) Training and validation Accuracy of the custom model, (b) Training and validation loss of a custom model.

Figure 8 (a) shows the model accuracy for our custom model as it improves with the successive epochs, and figures 8 (b) shows the training loss for our custom model as it reduces with the consecutive epochs.

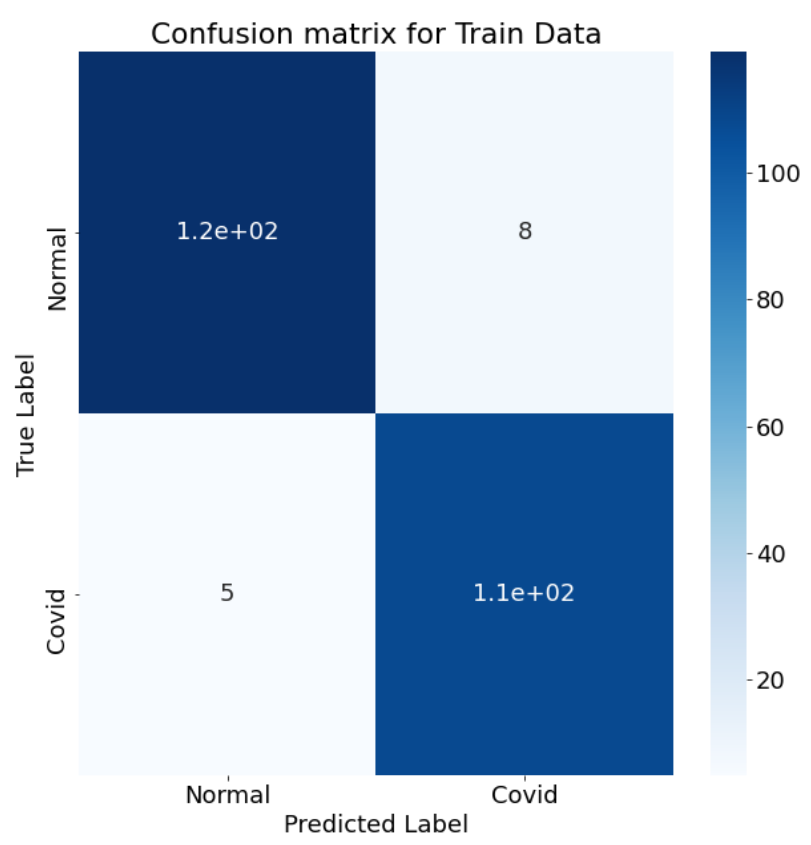

(a)

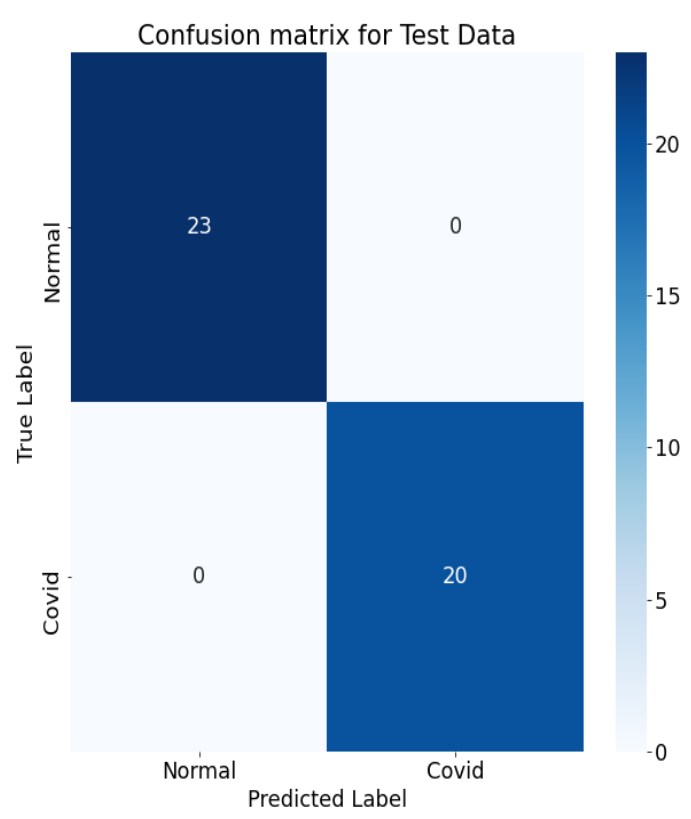

(b)

Figure 9: Confusion matrix of (a) train data (b) test data of custom model 
Figure 9 (a) and figure 9 (b) show the confusion matrices obtained for the train and test set, respectively. Figure 9 shows that the misclassified samples are negligible.

\section{Conclusion and Future Scope}

Covid-19 cases are rising daily, which poses a new challenge for the healthcare system to develop fast and reliable testing methods. We have attempted to research about one such way by using CNN models to classify the coronavirus $\mathrm{X}$-ray images and have obtained satisfactory results. However, we do not aim to use these results to test COVID cases in real life. The actual performance of this model needs to be evaluated via medical research. Our purpose of this research is to find out possible ways of testing, which are efficient and fast. We have seen here that our VGG 19 model performs the best among the three given models and may be used in the future in real-time. In the future, we can collect medical images data for improving the performance of a model and fine-tuning as needed.

\section{References}

[1]. Huang, C., Wang, Y., Li, X., Ren, L., Zhao, J., Hu, Y., ... \& Cheng, Z. (2020). Clinical features of patients infected with 2019 novel coronavirus in Wuhan, China. The lancet, 395(10223), 497-506.

[2]. Apostolopoulos, I. D., \& Mpesiana, T. A. (2020). Covid-19: automatic detection from xray images utilizing transfer learning with convolutional neural networks. Physical and Engineering Sciences in Medicine, 1.

[3]. Basu, S., \& Mitra, S. (2020). Deep Learning for Screening COVID-19 using Chest X-Ray Images. arXiv preprint arXiv:2004.10507.

[4]. Hassan, M. (2019). VGG16: Convolutional Network for Classification and Detection.

[5]. Mateen, M., Wen, J., Song, S., \& Huang, Z. (2019). Fundus image classification using VGG-19 architecture with PCA and SVD. Symmetry, $11(1), 1$.

[6]. Townsend, J. T. (1971). Theoretical analysis of an alphabetic confusion matrix. Perception \& Psychophysics, 9(1), 40-50.

[7]. Joseph Paul Cohen and Paul Morrison and Lan DaoCOVID-19 image data collection, arXiv:2003.11597, https://github.com/ieee8023/covid- chestxraydataset

[8]. Paul Mooney, Chest X-ray Images (Pneumonia), accessed from: https://www.kaggle.com/paultimothymooney/c hest-xray-pneumonia.

[9]. Simonyan, K., \& Zisserman, A. (2014). Very deep convolutional networks for large-scale image recognition. arXiv preprint arXiv: 1409.1556.

[10]. Hall, L. O., Paul，R., Goldgof, D. B., \& Goldgof, G. M. (2020). Finding covid-19 from chest $\mathrm{x}$-rays using deep learning on a small dataset. arXiv preprint arXiv:2004.02060.

[11]. Bernheim, A., Mei, X., Huang, M., Yang, Y., Fayad, Z. A., Zhang, N., ... \& Li, S. (2020). Chest CT findings in coronavirus disease-19 (COVID-19): relationship to duration of infection. Radiology, 200463.

[12]. World Health Organization. (2020). Coronavirus disease 2019 ((19-COVID : situation report, 88.

[13]. Narin, A., Kaya, C., \& Pamuk, Z. (2020). Automatic detection of coronavirus disease (covid-19) using x-ray images and deep convolutional neural networks. arXiv preprint arXiv:2003.10849.

[14]. Borghesi, A., \& Maroldi, R. (2020). COVID19 outbreak in Italy: experimental chest X-ray scoring system for quantifying and monitoring disease progression. La radiologia medica, 1.

[15]. Pereira, R. M., Bertolini, D., Teixeira, L. O., Silla Jr, C. N., \& Costa, Y. M. (2020). COVID-19 identification in chest X-ray images on flat and hierarchical classification scenarios. Computer Methods and Programs in Biomedicine, 105532.

[16]. Afshar, P., Heidarian, S., Naderkhani, F., Oikonomou, A., Plataniotis, K. N., \& Mohammadi, A. (2020). Covid-caps: A capsule network-based framework for identification of covid-19 cases from x-ray images. arXiv preprint arXiv:2004.02696.

[17]. Ozturk, T., Talo, M., Yildirim, E. A., Baloglu, U. B., Yildirim, O., \& Acharya, U. R. (2020). Automated detection of COVID-19 cases using deep neural networks with X-ray images. Computers in Biology and Medicine, 103792.

[18]. Punn, N. S., \& Agarwal, S. (2020). Automated diagnosis of COVID-19 with limited posteroanterior chest X-ray images 
using fine-tuned deep neural networks. arXiv preprint arXiv:2004.11676.

[19]. Abbas, A., Abdelsamea, M. M., \& Gaber, M. M. (2020). Classification of COVID-19 in chest X-ray images using DeTraC deep convolutional neural network. arXiv preprint arXiv:2003.13815.

[20]. Karim, M., Döhmen, T., RebholzSchuhmann, D., Decker, S., Cochez, M., \& Beyan, O. (2020). Deepcovidexplainer: Explainable covid-19 predictions based on chest $\mathrm{x}$-ray images. arXiv preprint arXiv:2004.04582.

[21]. Minaee, S., Kafieh, R., Sonka, M., Yazdani, S., \& Soufi, G. J. (2020). Deep-covid: Predicting covid-19 from chest $\mathrm{x}$-ray images using deep transfer learning. arXiv preprint arXiv:2004.09363.

[22]. Li, X., Li, C., \& Zhu, D. (2020). COVIDMobileXpert: On-Device COVID-19 Screening using Snapshots of Chest X-Ray. arXiv preprint arXiv:2004.03042. 